\title{
Efficacy and safety of iodine- 125 brachytherapy combined with chemotherapy in the treatment of advanced NSCLC in the elderly
}

This article was published in the following Dove Press journal:

OncoTargets and Therapy

\section{Chunrong $\mathrm{Wu}^{1, *}$ \\ Bo $\mathrm{Li}^{2, *}$ \\ Guiyin Sun' \\ Chunfang Peng' \\ Debing Xiang'}

'Department of Oncology, Jiangjin

Center Hospital of Chongqing, Jiangjin, China; ${ }^{2}$ Department of

Cardiology, Jiangjin Center Hospital

of Chongqing, Jiangjin, China

*These authors contributed equally to this work
Correspondence: Debing Xiang

Department of Oncology, Jiangjin

Center Hospital of Chongqing,

725 Jiangzhou Avenue, Dingshan street, Jiangjin 402260, China

Tel +862347557382

Email xdb86@hotmail.com
Background: Advanced non-small-cell lung cancer (NSCLC) is a huge challenge for physicians. Traditional chemoradiotherapy is associated with high rates of toxicities, especially when treating gerontal patients. Our study was focused on investigating the safety and efficacy of permanent iodine-125 seed implantation and chemotherapy for the treatment of advanced NSCLC in the elderly.

Methods: Fifty elderly patients with stage III or IV NSCLC at our hospital from January 2011 to June 2017 were treated with the chemotherapy regimens (paclitaxel/cisplatin) and computed tomography (CT)-guided iodine-125 brachytherapy (group A), 50 patients who received chemotherapy consisting of paclitaxel and cisplatin only (group B) were matched-up with the patients in group A. The local response rate was evaluated by CT. Progression-free survival (PFS) and overall survival (OS) data were obtained through clinical follow-up.

Results: The patients were followed-up for 3-46 months. With a median follow-up time of 20 months, the OS and PFS were 20 months (95\% CI: 19.09-20.90 months) vs 15 months (95\% CI: $14.48-15.51$ months $)(P<0.05)$ and 13 months (95\% CI: $11.96-14.04$ months) vs 8 months (95\% CI: 7.63-8.37 months) $(P<0.05)$ in group A and group B, respectively. The symptoms of patients in group A were significantly relieved when compared with group B. Severe complications were not observed in either of the groups.

Conclusion: The combination of iodine-125 seed brachytherapy and chemotherapy is an effective and safe therapy and is superior to chemotherapy alone for advanced NSCLC in the elderly.

Keywords: iodine-125, brachytherapy, chemotherapy, elderly, NSCLC

\section{Introduction}

Lung cancer (LC) has become a major malignancy worldwide, and it has been the primary cause of cancer-related mortality in recent decades. ${ }^{1}$ Approximately $80 \%$ of LCs are classified as advanced non-small-cell lung cancer (NSCLC), which is the most common form of LC. Most NSCLC patients are diagnosed when the disease is at the extremely late stage, when it has metastasized to the liver, bone or brain, especially in elderly patients. The WHO chronologically classified old age into three categories as follows: middle aged (45-59 years old), elderly (60-74 years old), and advanced age (75 years or older). ${ }^{2}$ Therefore, patients aged $\geq 60$ years are considered to be elderly patients. Elderly LC patients are characterized by poor short-term deterioration and rapid decline of general condition, ie, cough, chest pain, dyspnea, and weight loss. Currently, the standard treatments available, including surgery, chemotherapy, and radiation, are far from being satisfactory when treating elderly patients. Although chemoradiotherapy is recommended for subsequent treatment, it is associated with higher rates of toxicities, 
including radiation pneumonitis, radiation esophagitis, and tracheal necrosis. ${ }^{3}$ Particularly in the elderly, either from continuous damage caused by long-term smoking or any other physiologic reason, they easily develop pneumonia, pulmonary blisters, COPD, and poor general condition. A series of clinical trials indicated that quality of life (QOL) has been considered an all-important end-point for clinical cancer research, especially in elderly patients with NSCLC. ${ }^{4}$

The physical characteristics of the iodine-125 seed include small size, low-energy, and short half-life decay time. Generally, the treatment time does not exceed 30-45 minutes. ${ }^{5}$ The permanent implantation of the iodine-125 seed is thought to have the advantage of releasing a high radiation dose (100-140 Gy) to a tumor mass, and this dose is sharply reduced within a short distance. To a large extent, the risk of serious radiationinduced pneumonitis is reduced. ${ }^{6}$ Additionally, other toxicities such as hematologic toxicities, esophagitis, mucositis, and radiodermatitis are rarely reported, and the incidence of complications of chemotherapy is not increased. ${ }^{7}$ It is promising that patients showed better adherence because of the short treatment time. Currently, iodine-125 seed implantation has been successfully used for the treatment of advanced and refractory LC. ${ }^{7-10}$ However, this therapy has been rarely reported for treating elderly patients with advanced NSCLC.

In recent years, we have performed iodine-125 seed implantation therapy on a large number of elderly patients with NSCLC in our oncology department. The purpose of this retrospective study was to evaluate the efficacy and safety of combination of iodine-125 seed implantation and chemotherapy for elderly patients with advanced NSCLC. Meanwhile, the QOL of patients undergoing computed tomography (CT)-guided iodine-125 brachytherapy was still under discussion.

\section{Materials and methods}

\section{Patients}

One hundred patients with stage III or IV NSCLC were enrolled from January 2011 to June 2017. The diagnosis of all cases was confirmed by needle biopsy or bronchoscopic biopsy. Pathologists confirmed the malignant morphology based on H\&E examination, immunohistochemical staining, and molecular studies. Imaging such as CT or positron emission tomography-CT was used primarily for detection and staging. The clinical characteristics of these patients were listed in Table 1. The TNM staging of the tumors was performed according to the American Joint Committee on Cancer seventh Edition. ${ }^{11}$ The general condition of the patients was acceptable (lifespan longer than 3 months, sufficient hematopoietic and hepatorenal function, normal coagulation function), and the mean age was 68 years (range, 60-79 years). Patients in
Table I Clinical features of patients enrolled in this study

\begin{tabular}{|c|c|c|c|}
\hline Characteristics & $\begin{array}{l}\text { Group A } \\
(n=50)\end{array}$ & $\begin{array}{l}\text { Group B } \\
(n=50)\end{array}$ & $P$-value \\
\hline Age (average, scale) & $68(60-76)$ & $68(60-79)$ & 0.986 \\
\hline \multicolumn{4}{|l|}{ Gender } \\
\hline Male & 38 & 35 & 0.653 \\
\hline Female & 12 & 15 & \\
\hline \multicolumn{4}{|l|}{ Pathology } \\
\hline Adenocarcinomas & 23 & 26 & 0.689 \\
\hline Squamous cell carcinomas & 27 & 24 & \\
\hline \multicolumn{4}{|l|}{ Stage } \\
\hline III & 17 & 14 & 0.666 \\
\hline IV & 33 & 36 & \\
\hline \multicolumn{4}{|l|}{ Pain score } \\
\hline 0 & 6 & 8 & 0.78 \\
\hline $1-3$ & 18 & 19 & \\
\hline $4-6$ & 26 & 23 & \\
\hline $7-10$ & 0 & 0 & \\
\hline \multicolumn{4}{|l|}{ ECOG score } \\
\hline $0-1$ & 16 & 14 & 0.828 \\
\hline 2 & 34 & 36 & \\
\hline
\end{tabular}

Notes: Group A, 50 elderly patients with stage III or IV NSCLC treated with the chemotherapy regimens (paclitaxel/cisplatin) and CT-guided iodine- 125 brachytherapy. Group B, 50 patients who received chemotherapy consisting of paclitaxel and cisplatin only.

Abbreviations: $\mathrm{CT}$, computer tomography; ECOG, Eastern Cooperative Oncology Group; NSCLC, non-small-cell lung cancer.

group A (50 elderly patients with stage III or IV NSCLC treated with the chemotherapy regimens [paclitaxel/cisplatin] and CT-guided iodine-125 brachytherapy) were excluded from the study if they met the following criteria: refusal of iodine-125 seed implantation treatment, or not suitable for iodine-125 seed implantation treatment (tumors near the heart, large blood vessels, spinal cord, or the mediastinum). All the patients received chemotherapy, and patients in group A underwent iodine-125 implantation 2 weeks after the first cycle of chemotherapy. The research protocol was approved by the Ethics Committee of the Jiangjin Center Hospital of Chongqing, and written informed consent was received from each patient.

\section{Chemotherapy}

All the patients received chemotherapy consisting of paclitaxel $\left(135 \mathrm{mg} / \mathrm{m}^{2}\right.$ on day 1$)$ and cisplatin $\left(30 \mathrm{mg} / \mathrm{m}^{2}\right.$ on days 1 , 2 , and 3). The chemotherapy regimen had a 21-day schedule, and the schedule was repeated for 4-6 cycles in both group A and $\mathrm{B}$ patients, if tolerated. Routine blood examination and coagulation function, liver and kidney function tests were performed, and enhanced-CT scans were also performed every 3 months to evaluate the tumor volume.

\section{CT-guided implantation of iodine- 125 seeds}

A CT scanner (Siemens Somatom Sensation 64 CT Scanner) was applied to calculate the tumor margin and implantation of 
iodine-125 seeds. The radiotherapy (RT) treatment planning system (TPS; HGGR-2000) was provided by Beijing Feitian Zhaoye Technology Development Co. LTD (Beijing, China). The preoperative TPS was used for precise localization of the radioactive seed implantation. The RT physicians formulated the planning target volume dose of 100-140 Gy. Under CT guidance, the implantation needles accurately reached the tumor without injuring vital organs such as the major bronchi, vital blood vessels, heart, and spinal cord. The iodine-125 seeds were released by needles at an interval of $1-1.5 \mathrm{~cm}$. After the operation, CT was used to examine whether there were complications related to implantation (eg, bleeding, seed migration, and pneumothorax). Next, the patients were kept in the radiation oncology ward, and a piece of lead towel covered the surgical wound.

\section{Evaluation of efficacy and safety}

The efficacy and safety were evaluated 6 months after treatment. The tumor response was evaluated according to the Response Evaluation Criteria in Solid Tumors criteria. ${ }^{12}$ The response rate (RR) was calculated as (complete response $[\mathrm{CR}]+$ partial response $[\mathrm{PR}]$ )/total number of patients $\times 100 \%$. The progression-free survival (PFS) time was based on the time interval from the start of treatment to disease progression, death, or end of the study. The overall survival (OS) time was the time interval from start of treatment to death. The toxicity criteria of the Radiation Therapy Oncology Group (RTOG) were applied to assess the acute and late adverse effects of irradiation. The pain score was calculated using the numerical rating scale: 0 points indicated no pain, 1-3 points indicated mild pain, 4-6 points indicated moderate pain, and 7-10 indicated severe pain. ${ }^{13}$ The Eastern Cooperative Oncology Group (ECOG) performance status of patients was calculated as follows: 0 points indicated fully active and completely normal, one point indicated freedom of movement and light physical activity, two points indicated patient was awake more than half the day, three points indicated patient spent more than half the day in bed or in a wheelchair, four points indicated a bedridden status - patient was not self-reliant, and five points indicated death. ${ }^{14}$

\section{Follow-up}

All patients were followed-up at 3 months following chemotherapy with or without seed implantation, and then every 3 months for up to 46 months, with a median of 20 months. Routine laboratory examination and imaging examination were performed to identify possible complications. The patients' blood cell count, liver and renal function, pain score, radiation-induced pneumonia status, $R R$, progressive-free time, and death time were recorded in detail.

\section{Statistical analysis}

Data processing and analysis were performed using SPSS version 17.0 for Windows. Continuous data were expressed as mean \pm SD and were determined by performing Student's $t$-test. Categorical variables were analyzed using $\chi^{2}$ test or Fisher's exact test. The survival curves of patients in the two groups were analyzed using the Kaplan-Meier method. $P<0.05$ was considered statistically significant difference.

\section{Results \\ Patient characteristics}

The baseline characteristics of the two groups were well-balanced in terms of age, gender, pathology, tumor stage, ECOG status, and pain score (Table 1). All the patients in group A successfully tolerated brachytherapy with iodine-125 seed implantation.

\section{Relief of clinical symptoms}

Compared with group B (50 elderly patients with stage III or IV NSCLC treated with chemotherapy consisting of paclitaxel and cisplatin only), the symptoms of patients in group A were significantly relieved after treatment, especially regarding pain relief (Table $2, P=0.025$ ) and ECOG scores (Table 3, $P=0.036$ ).

\section{RR and survival outcomes}

The median follow-up time was 20 months (range: 3-46 months). After the 6-month follow-up of patients in both groups, CR and PR were observed in 4/50 (8\%) and 32/50 (64\%) patients in group A and 0/50 (0.0\%) and 25/50 (50\%) in group B (Figure 1), and 2/50 patients with progressive disease (4\%) in group A vs 7/50 patients (14\%) in group B. The RR of the tumors of patients in group A was 36/50 (72\%) vs $25 / 50(50 \%)$ in group $\mathrm{B}\left(\chi^{2}=8.837 ; P=0.032\right.$; Table 4$)$.

The median PFS of patients was 13 months $(95 \% \mathrm{CI}$ : 11.96-14.04) in group A and 8 months (95\% CI: 7.63-8.37) in group $B$ (log-rank test, $\chi^{2}=118.47, P=0.000$; Figure 2). The median OS time was 20 months (95\% CI: 19.09-20.90) in

Table 2 Analysis of pain in patients after treatments

\begin{tabular}{l|l|l|l|l|l}
\hline Group & \multicolumn{4}{l|}{ Pain score } & P-value \\
\hline & 0 & $1-3$ & $4-6$ & $7-10$ & \\
A & 19 & 18 & 11 & 2 & \\
B & 10 & 13 & 19 & 8 & 0.025 \\
\hline
\end{tabular}

Notes: Group A, 50 elderly patients with stage III or IV NSCLC treated with the chemotherapy regimens (paclitaxel/cisplatin) and CT-guided iodine- 125 brachytherapy. Group B, 50 patients who received chemotherapy consisting of paclitaxel and cisplatin only.

Abbreviations: CT, computer tomography; NSCLC, non-small-cell lung cancer. 
Table 3 Analysis of physical status score in patients after treatments

\begin{tabular}{l|l|l|l|l|l}
\hline Group & \multicolumn{4}{l|}{ ECOG score } & P-value \\
\hline & $0-1$ & 2 & 3 & 4 & \\
A & 25 & 21 & 4 & 0 & \\
B & 18 & 17 & 12 & 3 & 0.036 \\
\hline
\end{tabular}

Notes: Group A, 50 elderly patients with stage III or IV NSCLC treated with the chemotherapy regimens (paclitaxel/cisplatin) and CT-guided iodine- 125 brachytherapy. Group B, 50 patients who received chemotherapy consisting of paclitaxel and cisplatin only.

Abbreviations: $\mathrm{CT}$, computer tomography; Eastern Cooperative Oncology Group; NSCLC, non-small-cell lung cancer.
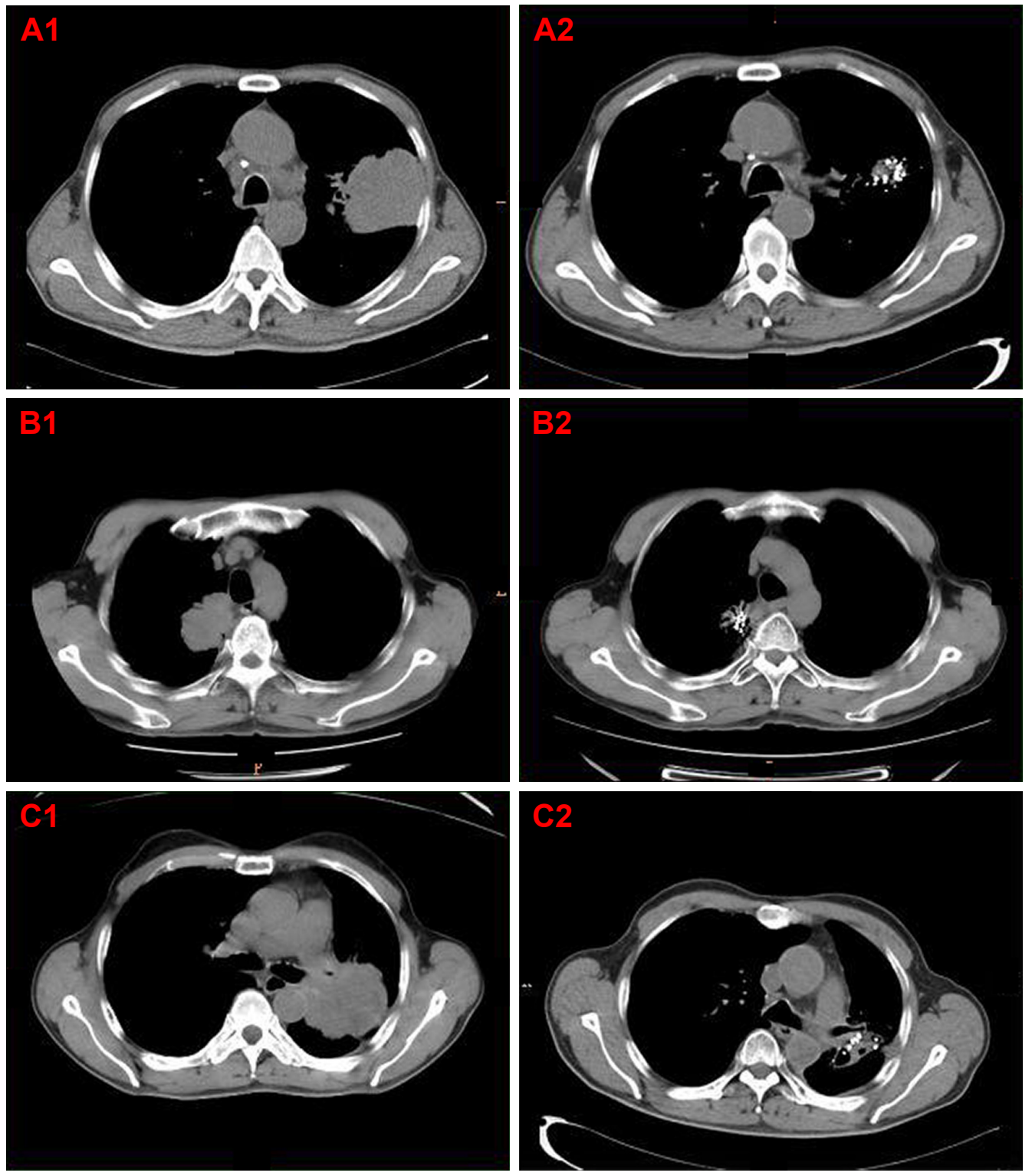

Figure I CT scan images of typical patients in group $A$.

Notes: (AI, A2) CT scan of a 62-year-old male patient with adenocarcinoma. (AI) The pre-treatment CT image shows a mass in the left upper lobe (October I4, 20I3). (A2) The target lesion completely disappeared after the combination of iodine- 125 seed implantation and chemotherapy regimen. The chest pain symptoms were relieved considerably (March 12, 20I4). (B I, B2). CT scan of a 60-year-old male patient with adenocarcinoma. (B I) The pre-treatment lung CT image shows a mass near the trachea in the right upper lobe (October 8, 2016). (B2) Six months after treatment, the CT scan indicated a complete response (April I7, 20I7). (CI, C2) CT scan of a 67-year-old male patient with squamous cell carcinoma. (CI) The pre-treatment CT image shows an irregular mass in the left lower lobe (June I, 20I6). (C2) The lesion is significantly decreased in size and partially cavitated 6 months after treatment. Difficult breathing was relieved obviously (December 5, 20I6). Group A, 50 elderly patients with stage III or IV NSCLC treated with the chemotherapy regimens (paclitaxel/cisplatin) and CT-guided iodine- 125 brachytherapy.

Abbreviations: CT, computer tomography; NSCLC, non-small-cell lung cancer. 
Table 4 Comparison of treatment effect in both groups

\begin{tabular}{l|l|l|l|l|l|l}
\hline Group & CR & PR & SD & PD & RR $=$ CR+PR(\%) & P-value \\
\hline A & 4 & 32 & 12 & 2 & $36(72 \%)$ & 0.032 \\
B & 0 & 25 & 18 & 7 & $25(50 \%)$ & \\
\hline
\end{tabular}

Notes: Group A, 50 elderly patients with stage III or IV NSCLC treated with the chemotherapy regimens (paclitaxel/cisplatin) and CT-guided iodine- 125 brachytherapy. Group B, 50 patients who received chemotherapy consisting of paclitaxel and cisplatin only.

Abbreviations: CT, computer tomography; NSCLC, non-small-cell lung cancer; $R R$, response rate; $C R$, complete response ; $S D$, stable disease; $P R$, partial response; $\mathrm{PD}$, progressive disease.

Hematological toxicity was defined as leukocyte decrease, anemia, and platelet decrease. No statistically significant difference was found in the hematological toxic events between group A and group B (Table 6, $P>0.05$ ). Meanwhile, severe liver toxicity was not observed in either of the two groups (eight of 50 patients [16\%] in group A vs six of 50 patients $[12 \%]$ in group $B, P=0.790$ ) (Table 6). Severe complications of grade 4 or above did not occur.

\section{Discussion}

LC is characterized by high morbidity and seriously threatens human life and health. Most LC patients are diagnosed at advanced stage and cannot undergo surgical resection. Thus, chemoradiotherapy is recommended for these patients. It is unsatisfactory that chemotherapy with cytotoxic drugs has achieved very small survival benefits and is encumbered by significant toxicity such as myelosuppression, nausea and vomiting, liver and kidney function damage, cardiotoxicity, skin pigmentation, and hair loss. External irradiation is usually regarded as

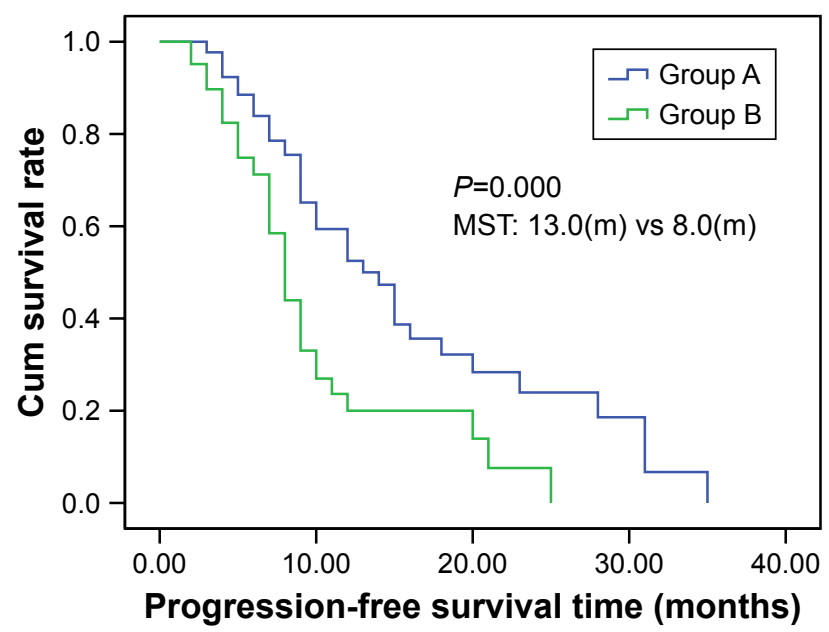

Figure 2 Kaplan-Meier curve of progression-free survival of patients in groups A and $\mathrm{B}$.

Notes: Group A, 50 elderly patients with stage III or IV NSCLC treated with the chemotherapy regimens (paclitaxel/cisplatin) and CT-guided iodine- 125 brachytherapy. Group B, 50 patients who received chemotherapy consisting of paclitaxel and cisplatin only.

Abbreviations: CT, computer tomography; cum, cumulative; NSCLC, non-smallcell lung cancer.

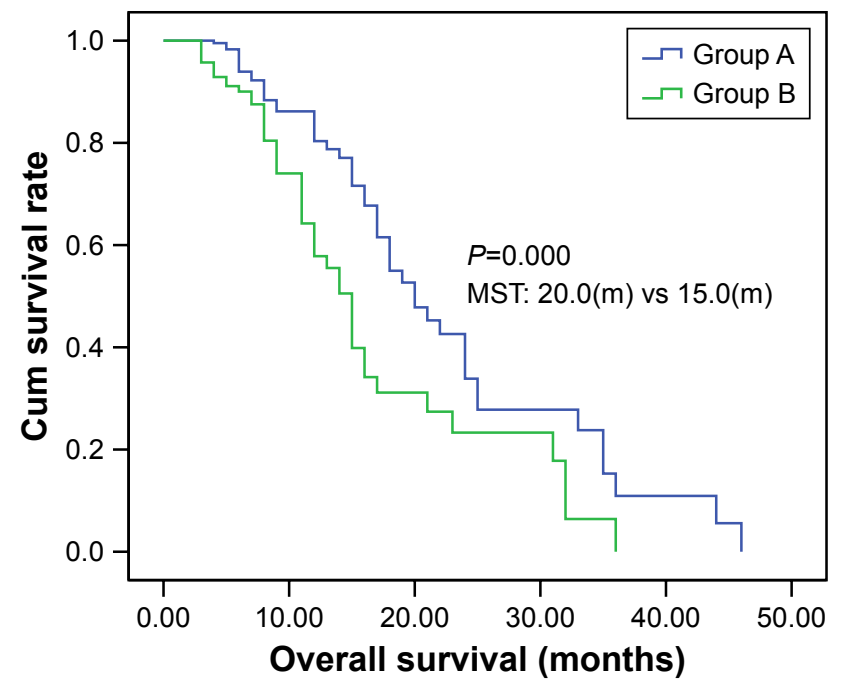

Figure 3 Kaplan-Meier curve of overall survival of patients in groups A and B. Notes: Group A, 50 elderly patients with stage III or IV NSCLC treated with the chemotherapy regimens (paclitaxel/cisplatin) and CT-guided iodine- 125 brachytherapy. Group B, 50 patients who received chemotherapy consisting of paclitaxel and cisplatin only.

Abbreviations: CT, computer tomography; cum, cumulative; NSCLC, non-smallcell lung cancer.

standard treatment but is associated with more serious side effects, such as radiation pneumonitis and esophagitis. In addition, the RT period is generally more than 1 month, greatly affecting the patient's treatment compliance. Elderly patients with LC usually suffer from poor lung function (eg, chronic obstructive emphysema) and poor general condition. QOL assessment is of great concern in clinical cancer research dedicated to elderly patients. The MILES trial has shown that QOL was one of the factors that impact whether combination chemotherapy or single drug therapy is used for elderly NSCLC patients. ${ }^{15}$ However, proper treatment strategies for elderly patients are far from convincing. Therefore, NSCLC in elderly patients is considered a model in the field of tumor research, and attention should be focused on QOL evaluation.

It was demonstrated in the RTOG 94-10 trial that several common toxicities of concurrent chemoradiotherapy in patients with local advanced (LA)-NSCLC specifically, the acute toxicities of radiation pneumonitis

Table 5 Complications after brachytherapy with iodine- 125 seed

\begin{tabular}{l|l|l}
\hline Complications & Group A & Group B \\
\hline Mild pneumothorax & 4 & 0 \\
Radiation pneumonitis & 0 & 0 \\
Seed migration & 2 & 0 \\
Local skin erythema & 0 & 0 \\
\hline
\end{tabular}

Notes: Group A, 50 elderly patients with stage III or IV NSCLC treated with the chemotherapy regimens (paclitaxel/cisplatin) and CT-guided iodine- 125 brachytherapy. Group B, 50 patients who received chemotherapy consisting of paclitaxel and cisplatin only.

Abbreviations: CT, computer tomography; NSCLC, non-small-cell lung cancer. 
Table 6 Adverse events observed in both groups upon follow-up

\begin{tabular}{|c|c|c|c|c|c|c|c|c|c|c|c|c|c|}
\hline \multirow[t]{2}{*}{ Grade } & \multicolumn{6}{|c|}{ Group A } & \multicolumn{6}{|c|}{ Group B } & \multirow[t]{2}{*}{$P$-value } \\
\hline & 0 & 1 & 2 & 3 & 4 & 5 & 0 & $\mathbf{I}$ & 2 & 3 & 4 & 5 & \\
\hline \multicolumn{14}{|l|}{ Hematology toxicity } \\
\hline Leukocyte decrease & 19 & 18 & 9 & 4 & 0 & 0 & 20 & 17 & 8 & 5 & 0 & 0 & 0.974 \\
\hline Anemia & 17 & 26 & 6 & 1 & 0 & 0 & 15 & 25 & 7 & 3 & 0 & 0 & 0.748 \\
\hline Platelet decrease & 18 & 25 & 4 & 3 & 0 & 0 & 20 & 22 & 6 & 2 & 0 & 0 & 0.825 \\
\hline Liver function lesion & 42 & 6 & 2 & 0 & 0 & 0 & 44 & 5 & 1 & 0 & 0 & 0 & 0.790 \\
\hline
\end{tabular}

Notes: Group A, 50 elderly patients with stage III or IV NSCLC treated with the chemotherapy regimens (paclitaxel/cisplatin) and CT-guided iodine-I25 brachytherapy. Group B, 50 patients who received chemotherapy consisting of paclitaxel and cisplatin only.

Abbreviations: $\mathrm{CT}$, computer tomography; NSCLC, non-small-cell lung cancer.

and esophagitis - result in chemotherapy delay or chemotherapeutic drug reductions, late radiation-induced pulmonary fibrosis, and cardiac toxicities, significantly impacting the QOL of patients and even their survival time. ${ }^{16}$ Recent advances in new RT technologies, such as IMRT, ${ }^{17}$ IGRT, ${ }^{18,19}$ and SBRT, ${ }^{20}$ are associated with higher radiation doses and more conformal RT at the tumor site, while the radiation dose outside of the primary target is decreased rapidly. Better knowledge of supportive care has reduced the rates of RT-induced toxicities. ${ }^{21,22}$ A report from the M.D. Anderson Cancer Center noted that the use of proton beam therapy allowed the safe delivery of a higher radiation dose; however, the incidences of severe radiation pneumonitis and esophagitis were lower. ${ }^{23}$

As an internal RT, iodine-125 seed implantation has been successfully used to treat a series of malignant tumors, such as prostate cancer, ${ }^{24}$ brain tumors, ${ }^{25,26}$ pancreatic cancer, ${ }^{27}$ skin cancer, ${ }^{28}$ ovarian cancer, ${ }^{29}$ recurrent cervical cancer, ${ }^{30}$ and head and neck neoplasms, ${ }^{31}$ especially in NSCLC. Santos et al indicated that the use of iodine-125 brachytherapy after sublobar resection significantly improved local control compared with sublobar resection alone $(18.6$ vs $2 \% ; P<0.0001) .{ }^{32}$ Fernando et al reported resection plus brachytherapy vs wedge resection alone with a median follow-up of nearly 4.4 years and 222 evaluable patients, with a little less than $13 \%$ of patients developing local recurrence at 3 years. His research has led to surgeons focusing more on what should be considered an appropriate surgical margin despite sublobar surgery. ${ }^{33}$ Both of their studies showed that adjuvant intraoperative brachytherapy in combination with sublobar resection did not increase perioperative pulmonary complications or worsen pulmonary function. Li et al found that patients with multiple recurrence or metastatic stage III/IV NSCLC could obtain longer PFS time and higher OS rates when treated with iodine-125 seed brachytherapy. ${ }^{10}$ Yu et al explored the effectiveness of brachytherapy plus chemotherapy vs chemotherapy alone for recurrent stage III NSCLC (52 patients were equally divided into two groups), and the PFS was 8 months vs 5.5 months $(P<0.05)$ with a median follow-up time of 11 months, and no severe complications. ${ }^{34}$ In our study, the median PFS (13 months vs 8 months) and OS (20 months vs 15 months) time were significantly longer for the patients with iodine-125 seed implantation combined with chemotherapy than with chemotherapy alone (Figures 2 and 3). In addition, the combined treatment offered better tumor control (Figure 1 and Table 4). The longer PFS and OS, as well as better RR rate mean that iodine- 125 brachytherapy combined with chemotherapy was more effective when compared with chemotherapy alone. Our study showed that the PFS of group A was longer than that in Yu et al's research, it may be because our median follow-up time was longer (20 months), our patients were stage III or IV NSCLC, and our sample number was greater (100 patients divided into two groups).

Iodine-125 implantation has remarkable superiority in improving QOL and relieving tumor-related symptoms. Song et al elucidated that, compared with best supportive care, it is noteworthy that iodine- 125 brachytherapy not only improved the survival and QOL of patients with LA-NSCLC but also significantly relieved tumor-related symptoms, such as cough, chest pain, chest tightness, and hoarseness. ${ }^{35}$ Jiao et al explored the efficiency and safety of iodine- 125 seed brachytherapy for painful bone metastases caused by malignancy, and patients who received radiofrequency ablation treatment or iodine-125 implantation obtained good long-term pain relief, and the complications were lower, with no treatment-related mortality. Thus, iodine- 125 seeds can be used as a safe and effective method for patients who develop painful bone metastases. ${ }^{36}$ In our research, there was no severe complications, such as massive hemoptysis, radiation pneumonitis, severe pneumothorax, infection, hematological toxicity, liver or kidney function lesion, or death (Tables 5 and 6). Patients treated in group A were 
clearly better than those in group B regarding many aspects, such as health status/QOL, physical functioning, fatigue, and pain relief. In group A, we found obvious pain relief in 19 patients (Table 2), and the ECOG scores were improved in 25 patients (Table 3 ). In group $B$, the pain was significantly worse in eight patients, and three patients were bedridden. The better pain relief and ECOG scores mean that iodine125 brachytherapy combined with chemotherapy was more effective when compared with chemotherapy alone, and able to contribute to better QOL in elderly patients with advanced NSCLC.

There was no occurrence of severe complications during the study. In group A, no patient developed radiation pneumonitis, local skin erythema, or hemoptysis (Table 5). The patients with mild pneumothorax did not experience chest suppression, and the pneumothorax was gradually absorbed and required no further therapy. Some patients experienced toxicities such as asthenia, vomiting, and anorexia which were rapidly resolved after active supportive care. Hematological toxicity was infrequent, however, in group A, four patients had severe leukocyte decrease (grade 3 ), one patient had severe anemia (grade 3 ), and three patients had serious platelet decrease (grade 3 ). The hemocyte count was elevated following treatment with granulocyte colony-stimulating factor, thrombopoietin, recombinant human IL-11, and erythropoietin. In group B, five of 50 patients (10\%) showed severe leukocyte decrease (grade 3), while three patients $(6 \%)$ displayed severe anemia (grade 3 ) (Table 6). Our study proved that iodine-125 brachytherapy combined with chemotherapy was safe, and did not pose a greater risk to patients.

\section{Conclusion}

Our study preliminarily demonstrated that iodine-125 seed implantation combined with chemotherapy was effective and safe for advanced NSCLC in elderly patients, leading to fewer side effects, higher survival rate, and better QOL. Future research is needed to further confirm the efficacy and safety of this treatment.

\section{Acknowledgments}

We thank all participants and patients who participated in this study.

This study was supported by District Science and Technology Commission Project of Chongqing Jiangjin (grant number Y2017016), and Health and Family Planning Commission Medical Research Project of Chongqing (grant number 2017MSXM171).

\section{Author contributions}

Chunrong Wu designed the study and drafted the manuscript; Bo Li interpreted the data and performed statistical analysis; Guiyin Sun and Chunfang Peng participated in the coordination of the study; Debing Xiang supervised the study and helped to draft the manuscript. All authors contributed toward data analysis,drafting and critically revising the paper and agree to be accountable for all aspects of the work.

\section{Disclosure}

The authors report no conflicts of interest in this work.

\section{References}

1. Siegel RL, Miller KD, Jemal A. Cancer Statistics. CA: a cancer journal for clinicians. 2017;2017(67):7-30.

2. Özcan MF, Altınova S, Atan A. Treatment approaches to small renal masses in patients of advanced age ( $\geq 75$ years). Turk JUrol. 2018;44(4): 281-286.

3. Verma V, Simone CB, Werner-Wasik M. Acute and Late Toxicities of Concurrent Chemoradiotherapy for Locally-Advanced Non-Small Cell Lung Cancer. Cancers. 2017;9(9):120.

4. di Maio M, Perrone F. Quality of Life in elderly patients with cancer. Health Qual Life Outcomes. 2003;1:44.

5. Skowronek J. Brachytherapy in the treatment of lung cancer - a valuable solution. J Contemp Brachytherapy. 2015;7(4):297-311.

6. Reveiz L, Rueda JR, Cardona AF. Palliative endobronchial brachytherapy for non-small cell lung cancer. Cochrane Database Syst Rev. 2012 12:Cd004284.

7. Qiu H, Ji J, Shao Z, et al. The Efficacy and Safety of Iodine-125 Brachytherapy Combined with Chemotherapy in Treatment of Advanced Lung Cancer: A Meta-Analysis. J Coll Physicians Surg Pak. 2017;27(4):237-245.

8. Huo X, Huo B, Wang H, et al. Implantation of computed tomographyguided Iodine- 125 seeds in combination with chemotherapy for the treatment of stage III non-small cell lung cancer. J Contemp Brachytherapy. 2017;9(6):527-534

9. Chen Y, Li Y, Jia Y, et al. Bronchial artery chemoembolization combined with radioactive iodine-125 seed implantation in the treatment of advanced nonsmall cell lung cancer. J Cancer Res Ther. 2017;13(4): 636-641.

10. Li W, Dan G, Jiang J, et al. Repeated iodine-125 seed implantations combined with external beam radiotherapy for the treatment of locally recurrent or metastatic stage III/IV non-small cell lung cancer: a retrospective study. Radiat Oncol. 2016;11(1):119.

11. Edge SB, Compton CC. The American Joint Committee on Cancer: the 7th edition of the AJCC cancer staging manual and the future of TNM. Ann Surg Oncol. 2010;17(6):1471-1474.

12. Therasse P, Arbuck SG, Eisenhauer EA, et al. New guidelines to evaluate the response to treatment in solid tumors. JNCI: Journal of the National Cancer Institute. 2000;92(3):205-216.

13. Farčić N, Barać I, Pačarić S, Lovrić I, Ilakovac V. Acute Postoperative Pain in Trauma Patients - The Fifth Vital Sign. Open Access Maced J Med Sci. 2017;5(3):310-315.

14. de Kock I, Mirhosseini M, Lau F, et al. Conversion of Karnofsky Performance Status (KPS) and Eastern Cooperative Oncology Group Performance Status (ECOG) to Palliative Performance Scale (PPS), and the interchangeability of PPS and KPS in prognostic tools. $J$ Palliat Care. 2013;29(3):163-169.

15. Gridelli C, Perrone F, Gallo C, et al; MILES Investigators, et al. Chemotherapy for elderly patients with advanced non-small-cell lung cancer the Multicenter Italian Lung Cancer in the Elderly Study (MILES) phase III randomized trial. J Natl Cancer Inst. 2003;95(5):362-372. 
16. Curran WJ, Paulus R, Langer CJ, et al. Sequential vs. concurrent chemoradiation for stage III non-small cell lung cancer: randomized phase III trial RTOG 9410. J Natl Cancer Inst. 2011;103(19):1452-1460.

17. Koshy M, Malik R, Spiotto M, et al. Association between intensity modulated radiotherapy and survival in patients with stage III nonsmall cell lung cancer treated with chemoradiotherapy. Lung Cancer. 2017; 108:222-227.

18. Arns A, Blessing M, Fleckenstein J, et al. Phantom-based evaluation of dose exposure of ultrafast combined $\mathrm{kV}-\mathrm{MV}-\mathrm{CBCT}$ towards clinical implementation for IGRT of lung cancer. PLoS One. 2017; 12(11):e0187710.

19. Deek MP, Kim S, Yue N, et al. Modern radiotherapy using image guidance for unresectable non-small cell lung cancer can improve outcomes in patients treated with chemoradiation therapy. J Thorac Dis. 2016;8(9):2602-2609.

20. Eriguchi T, Takeda A, Sanuki N, et al. Stereotactic body radiotherapy for operable early-stage non-small cell lung cancer. Lung Cancer. 2017;109:62-67.

21. Edbrooke L, Aranda S, Granger CL, et al. Benefits of home-based multidisciplinary exercise and supportive care in inoperable non-small cell lung cancer - protocol for a phase II randomised controlled trial. BMC Cancer. 2017;17(1):663.

22. Aubin M, Vézina L, Verreault R, et al. Effectiveness of an intervention to improve supportive care for family caregivers of patients with lung cancer: study protocol for a randomized controlled trial. Trials. 2017;18(1):304.

23. Sejpal S, Komaki R, Tsao A, et al. Early findings on toxicity of proton beam therapy with concurrent chemotherapy for nonsmall cell lung cancer. Cancer. 2011;117(13):3004-3013.

24. Zuber S, Weiß S, Baaske D, et al. Iodine-125 seed brachytherapy for early stage prostate cancer: a single-institution review. Radiat Oncol. 2015;10:49.

25. Shahzadi S, Azimi P, Parsa K. Long-Term Results of stereotactic Brachytherapy (Temporary 125Iodine Seeds) for the Treatment of LowGrade Astrocytoma (Grade II). Iran Red Crescent Med J. 2013;15(1): 49-57.
26. Schwarz SB, Thon N, Nikolajek K, et al. Iodine-125 brachytherapy for brain tumours - a review. Radiat Oncol. 2012;7:30.

27. Xu K, Niu L, Mu F, Hu Y. Cryosurgery in combination with brachytherapy of iodine-125 seeds for pancreatic cancer. Gland Surg. 2013;2(2):91-99.

28. Ferreira C, Johnson D, Rasmussen K, et al. A novel conformal superficial high-dose-rate brachytherapy device for the treatment of nonmelanoma skin cancer and keloids. Brachytherapy. 2017;16(1):215-222.

29. Wang Y, Zhang W, Liu P, Guo Z, Ni H. Computed tomography-guided 125I seed interstitial implantation in the treatment of recurrent ovarian cancer. Int J Gynecol Cancer. 2014;24(8):1414-1419.

30. Han L, Li C, Wang J, et al. Iodine-125 radioactive seed tissue implantation as a remedy treatment for recurrent cervical cancer. J Cancer Res Ther. 2016;12(Supplement):C176-C180.

31. Wu WJ, Shao X, Huang MW, et al. Postoperative iodine-125 interstitial brachytherapy for the early stages of minor salivary gland carcinomas of the lip and buccal mucosa with positive or close margins. Head Neck. 2017;39(3):572-577.

32. Santos R, Colonias A, Parda D, et al. Comparison between sublobar resection and 125Iodine brachytherapy after sublobar resection in highrisk patients with Stage I non-small-cell lung cancer. Surgery. 2003; 134(4):691-697.

33. Fernando HC, Landreneau RJ, Mandrekar SJ, et al. The impact of adjuvant brachytherapy with sublobar resection on pulmonary function and dyspnea in high-risk patients with operable disease: preliminary results from the American College of Surgeons Oncology Group Z4032 trial. J Thorac Cardiovasc Surg. 2011;142(3):554-562.

34. Yu X, Li J, Zhong X, He J. Combination of Iodine-125 brachytherapy and chemotherapy for locally recurrent stage III non-small cell lung cancer after concurrent chemoradiotherapy. BMC Cancer. 2015;15:656.

35. Song J, Fan X, Zhao Z, et al. ${ }^{125}$ I brachytherapy of locally advanced non-small-cell lung cancer after one cycle of first-line chemotherapy: a comparison with best supportive care. Onco Targets Ther. 2017; 10:1345-1352.

36. Jiao D, Wu G, Ren J, Han X. Radiofrequency ablation versus $125 \mathrm{I}$-seed brachytherapy for painful metastases involving the bone. Oncotarget. 2016;7(52):87523-87531
OncoTargets and Therapy

\section{Publish your work in this journal}

OncoTargets and Therapy is an international, peer-reviewed, open access journal focusing on the pathological basis of all cancers, potential targets for therapy and treatment protocols employed to improve the management of cancer patients. The journal also focuses on the impact of management programs and new therapeutic agents and protocols on

\section{Dovepress}

patient perspectives such as quality of life, adherence and satisfaction The manuscript management system is completely online and includes a very quick and fair peer-review system, which is all easy to use. Visit http://www.dovepress.com/testimonials.php to read real quotes from published authors. 